\title{
Significance of new membrane formation in peritoneal biopsies of peritoneal dialysis patients: a case-control study
}

Kazuho Honda ${ }^{1 *}$, Chieko Hamada ${ }^{2}$, Kunio Kawanishi ${ }^{3}$, Masaaki Nakayama ${ }^{4}$, Masanobu Miyazaki ${ }^{5}$, Yasuhiko Ito ${ }^{6}$ and on behalf of the Peritoneal Pathology Study Committee of Japanese Society of Peritoneal Dialysis (JSPD) ${ }^{7}$

\begin{abstract}
Background: Newly formed membrane (NFM) on the peritoneal membrane proper is a unique pathological hallmark of encapsulating peritoneal sclerosis (EPS), but its definition and diagnostic significance have not been well described. This study investigated the pathological features of NFM in EPS and prevalence of NFM in peritoneal biopsy at catheter removal.

Methods: This multicenter retrospective, observational study was conducted by the Japanese Society of Peritoneal Dialysis and enrolled ten patients with and 52 without EPS at peritoneal biopsy during enterolysis surgery or catheter removal. All patients were treated using conventional, acidic peritoneal dialysis (PD) solutions. Thirty of the 52 non-EPS patients perform peritoneal lavage once daily after completing PD to prevent the development of EPS and 22 discontinued PD without lavage. NFM was defined as additional membrane structure on the peritoneum that is properly characterized by exudative or fibrous matrices and fibroblast-like cells. Immunostaining of fibrin and podoplanin was performed for the evaluation of NFM.

Results: NFM was confirmed histologically in eight of the ten patients with EPS. It was also detected in 13 of the 30 patients (43.3\%) in the post-PD lavage group and in one of the 22 patients (4.5\%) in the non-lavage group. The NFM histology showed various stages of EPS pathology, from early exudative changes with fibrin deposition (stage l; $n=5$ ), progressing to proliferative and fibrosing changes with podoplanin-positive fibroblast-like cells (stage II; $n=9$ ), and finally resulting in adhesive and fibrous scar formation (stage III; $n=8$ ). Immunostaining of fibrin and podoplanin was helpful for evaluating the stage of the NFM.

Conclusions: NFM contributes the encapsulation of intestines and is a pathological hallmark of EPS, but can be detected microscopically in patients without EPS, especially those with peritoneal lavage after PD. New membrane formation starts insidiously in peritoneal membrane damaged by PD treatment, but does not necessarily lead to the development of EPS.
\end{abstract}

Keywords: Encapsulating peritoneal sclerosis, Simple peritoneal fibrosis, Encapsulating membrane, Newly formed membrane, Fibrin, Podoplanin, Peritoneal permeability

\footnotetext{
* Correspondence: kzhonda@med.showa-u.ac.jp

'Department of Anatomy, Showa University School of Medicine, 1-5-8

Hatanodai, Shinagawa-ku, Tokyo 142-8555, Japan

Full list of author information is available at the end of the article
} 


\section{Background}

Encapsulating peritoneal sclerosis (EPS) is a rare but serious complication of peritoneal dialysis (PD) [1-4]. The diagnosis of EPS is based on clinical signs of obstructive ileus and pathological evidence of encapsulation of entire abdominal organs (cocoon formation) [5]. Histological evaluation of peritoneal tissues in EPS revealed that fibrin deposition, increased fibrosis, inflammatory cell infiltration, and angiogenesis were characteristic of EPS [6-9]; however, they were nonspecific inflammatory findings, and none were pathognomonic for the diagnosis of EPS. Macroscopic findings of abdominal organs suggested a newly formed encapsulating membrane, mainly in the visceral peritoneum, was unique to EPS [10]. Histologically, the structure of encapsulating membrane was described as organized fibrosing tissue formed on the peritoneal membrane proper with swollen fibroblast-like cells immunohistochemically positive for podoplanin $[11,12]$. These observations suggested that a definite pathological diagnosis of EPS requires macroscopic and/or histological confirmation of an encapsulating membrane. However, we have occasionally observed a newly formed membrane (NFM) similar to the encapsulating membrane of EPS in peritoneal biopsy specimens obtained from non-EPS patients with peritoneal lavage after PD withdrawal. Peritoneal lavage is frequently performed in Japan, especially in long-term PD patients, to prevent the development of EPS. It is believed to prevent EPS by elimination of exudates that lead to the formation of encapsulating membranes [13, 14].

The purpose of this study was to investigate the pathological features of encapsulating membranes as a diagnostic criterion of EPS and to evaluate the prevalence and significance of NFMs in peritoneal biopsies obtained at catheter removal. We also sought to identify the factors associated with new membrane formation, focusing on the extent of simple peritoneal sclerosis induced by PD and the effects of peritoneal lavage. We found that encapsulating membranes were diagnostic for EPS but that NFMs were frequently observed microscopically in biopsies of patients with peritoneal lavage after PD discontinuation but did not develop EPS. The presence of microscopic NFMs in peritoneal biopsies at catheter removal indicated increased peritoneal permeability and exudation but did not predict the subsequent development of EPS.

\section{Methods}

\section{Patients}

The Peritoneal Biopsy Program in Japan was conducted from 1994 to 2006. A total of 1928 peritoneal biopsy samples were collected from 227 medical institutes. Of these, 569 were excluded because of uncertain clinical information. Of the remaining 1359 samples, 230 were obtained at the initiation of PD and 1129 were obtained after PD of $66.4 \pm 44.4$ months duration. Patients with peritonitis within 1 month before PD withdrawal $(n=360)$ were excluded, and 79 of the remaining 769 samples were from patients with medical records indicating that they had been diagnosed with EPS or suspected EPS. We confirmed the diagnosis of EPS based on the Japanese guidelines for EPS [5] and International Society for Peritoneal Dialysis statement [15]. Clinical signs and symptoms of gastrointestinal obstruction were an essential diagnostic criterion of EPS. Image findings suggesting peritoneal membrane thickening or encapsulation of abdominal organs, bowel obstruction, or cocoon formation were supportive findings for the diagnosis. To ensure the diagnosis of EPS, only peritoneal biopsy samples obtained at enterolysis surgery were selected for inclusion of the EPS group ( $n=10$, five men and five women; PD duration of $124.5 \pm 34.2$ months). Consequently, most biopsy samples of the EPS patients were obtained from the adhering visceral peritoneum.

We selected 52 non-EPS patients with a PD duration of more than 60 months as matched-duration controls for the EPS group. The non-EPS patients were divided into two groups by the performance of peritoneal lavage treatment after PD withdrawal. The peritoneal lavage group included 30 patients, 19 men and 11 women, 58.5 \pm 9.5 years of age, a PD duration of $98.4 \pm 33.7$ months, and a lavage duration of $12.8 \pm 5.1$ months. The nonEPS patients without peritoneal lavage included 22 patients, 13 men and nine women, $44.1 \pm 17.5$ years of age, and PD duration of $91.4 \pm 30.3$ months. All biopsy samples of non-EPS patients were taken at catheter removal from the parietal peritoneum. No patient in the non-EPS groups, either with or without lavage, had clinical signs and symptoms of EPS. The reasons for PD discontinuation in the lavage group were ultrafiltration failure $(n=7)$, under-dialysis $(n=1)$, cerebral infarction $(n=1)$, EPS prevention $(n=2)$, and unknown $(n=19)$. Peritoneal lavage was usually performed once a day by exchange of PD solution with low glucose concentration $(n=25)$ or icodextrin $(n=5)$ solution for several months to years. The mean duration of peritoneal lavage was12.8 \pm 5.1 months. The clinical status of the nonlavage group varied, including patients who stopped PD because of ultrafiltration failure $(n=6)$, transplantation $(n=4)$, under-dialysis $(n=1)$, catheter-related problems $(n=3)$, malignancy $(n=2)$, and unknown $(n=1)$. Other patients were biopsied at catheter exchange $(n=4)$ or on exploratory laparotomy for the evaluation of peritoneal condition $(n=1)$. All patients in the EPS and non-EPS groups were treated using conventional acidic PD solutions. The study design was approved by the ethics committee of Juntendo Medical University (No. 370, May 2009). Written informed consent was obtained from all study subjects. 


\section{Histopathological analysis}

Peritoneal biopsy specimens were cut from the parietal peritoneum using a scalpel or peeled from the encapsulated visceral peritoneum and processed by routine histological procedures as previously described [16]. The adequacy of specimens for histological evaluation was determined in terms of size, site, and direction of the samples as described in the previous report [16]. All specimens included in the study were regarded as adequate for the evaluation of the peritoneal thickness, vasculopathy at post-capillary venules (PCVs), and presence or absence of NFM and its histological features. Two pathologists (KH and $\mathrm{KK})$ reviewed the samples together to arrive at a consensus evaluation. The extent of fibrosis and vascular sclerosis was evaluated as previously described [16]. In brief, to evaluate peritoneal fibrosis, we calculated average thickness $(\mu \mathrm{m})$ at five random points within the submesothelial compact zone (SMC) between the basal border of the surface mesothelial cells and upper border of the peritoneal adipose tissue. To evaluate vascular sclerosis, average ratio of lumen to vessel diameter was calculated at three to five randomly selected PCVs with external diameters ranging 25-50 $\mu \mathrm{m}$. NFMs were defined as formation of an additional membrane structure on the surface of the peritoneal membrane proper and comprised of fibrin exudation, collagenous fibrotic tissue, fibroblastlike cells, and capillary vessels. NFMs differ from peritoneal membrane proper, with simple peritoneal fibrosis and do not contain dense bundle of collagen, fascia, fat, or small arteries and veins that are seen in peritoneal tissue. The histological staging of NFMs included an early exudative phase with fibrin deposition and podoplanin-positive fibroblast-like cells (stage I), a proliferative and fibrosing phase with podoplanin-positive fibroblast-like cells but without fibrin deposition (stage II), and an adhesive and fibrous scarring phase without fibrin deposition or podoplanin-positive fibroblast-like cells (stage III). Immunostaining of fibrin and podoplanin was helpful for evaluating the stage of NFMs (Fig. 2j).

\section{Immunohistochemistry}

Mouse monoclonal anti-fibrin (MAB1901, Chemicon International) and human anti-podoplanin (\#11-003, AngioBio, Del Mar, CA, USA) were used as primary antibodies. The Envision+ System (Dako Cytomation) was used for detection of antibody binding and was visualized using diaminobenzidine tetrahydrochloride $(0.02 \%)$. Cell nuclei were counterstained with hematoxylin.

\section{Statistical analysis}

Data were reported as means \pm standard deviation. Differences were assessed for significance by $\chi^{2}$ tests, Student's $t$ tests, Mann-Whitney $U$ test, or KruskalWallis test, depending on whether the variables were categorical or continuous. Correlation coefficients were assessed for significance using the $F$ test. In all analyses, $p<0.05$ was considered significant.

\section{Results}

\section{Clinical and histological data of the ten EPS-developed} cases

The clinical and histological findings of each patient in the EPS group are shown in Table 1. The average PD duration was $124.5 \pm 34.2$ months. The biopsy samples were obtained during enterolysis surgery, which was performed at $23.6 \pm 19.2$ months after PD discontinuation to dissect intestinal adhesions. Peritoneal lavage was performed in three patients (no. 1, 3, and 4) for several months (7, 15, and 2 months, respectively) before enterolysis surgery to prevent or reduce abdominal adhesions. NFMs were identified microscopically in eight patients (80\%) and macroscopically during surgery in two

Table 1 Clinical and pathological characteristics of the EPS patients $(n=10)$

\begin{tabular}{|c|c|c|c|c|c|c|c|c|}
\hline Patient no. & Age & Sex & PD duration (m) & Post-PD duration (m) & Peritoneal lavage (m) & NFM (stage) & $\mathrm{SMC}(\mu \mathrm{m})$ & LN ratio \\
\hline 1 & 55 & $\mathrm{~F}$ & 79 & 15 & 7 & I & 389.2 & 0.352 \\
\hline 2 & 57 & M & 166 & 74 & - & III & 283 & 0.166 \\
\hline 3 & 68 & $\mathrm{~F}$ & 80 & 26 & 15 & I & 622 & 0.196 \\
\hline 4 & 56 & $\mathrm{~F}$ & 144 & 11 & 2 & I & 1269.6 & 0.186 \\
\hline 5 & 57 & M & 116 & 16 & - & I & 614.4 & 0.234 \\
\hline 6 & 60 & M & 151 & 35 & - & n.d. & 259.4 & 0.9 \\
\hline 7 & 56 & $\mathrm{~F}$ & 165 & 18 & - & ॥ & 389 & 0.192 \\
\hline 8 & 60 & $\mathrm{~F}$ & 142 & 18 & - & ॥ & 985.6 & 0.382 \\
\hline 9 & 52 & M & 84 & 9 & - & n.d. & 247 & 0.442 \\
\hline \multirow[t]{2}{*}{10} & 46 & M & 118 & 14 & - & I & 1302.8 & 0.263 \\
\hline & $56.7 \pm 5.7$ & $\begin{array}{l}\text { M5 } \\
\text { F5 }\end{array}$ & $124.5 \pm 34.2$ & $23.6 \pm 19.2$ & $\begin{array}{l}3 / 10(30 \%) \\
8.0 \pm 6.6\end{array}$ & $\begin{array}{l}8 / 10(80 \%) \\
{[5 / 2 / 1]}\end{array}$ & $636.2 \pm 409.4$ & $0.33 \pm 0.22$ \\
\hline
\end{tabular}


patients (20\%). In these two patients (no. 6 and 9), NFMs were not confirmed in the biopsy specimens. Histologically, most NFMs were stage I $(n=5)$, followed by stage II $(n=2)$, and stage III $(n=1)$, showing a predominance of early exudative stages in the encapsulating membrane formation. The stage III NFM was obtained from patient 2, who had the longest PD duration (166 months) and the longest post-PD duration (74 months). The extent of PD-related peritoneal sclerosis was evaluated by the thickness of the SMC and the lumen/vessel diameter ratio ( $\mathrm{L} / \mathrm{V}$ ratio) of the PCVs in the peritoneal membrane proper that was attached to the NFM in the biopsy specimens. The increased thickness of the SMC (636.2 \pm $409.4 \mu \mathrm{m})$ and the decreased $\mathrm{L} / \mathrm{V}$ ratio $(0.33 \pm 0.22)$ suggested a tendency of advanced peritoneal sclerosis in the EPS patients.

\section{Pathology of NFMs in EPS patients}

Figure 1 shows an NFM in an EPS patient (no. 4) obtained during enterolysis surgery. Microscopically, the NFM was identified as a fibrotic structure on the visceral peritoneum proper of the intestine (Fig. 1a). The thickness of the NFM was approximately 200-500 $\mu \mathrm{m}$ and characterized by interlacing homogenous fibrous bundles with fibroblastlike cells (Fig. 1b). Fibrin deposition was observed on the membrane surface by Masson trichrome staining (Fig. 1b) and confirmed by immunohistochemical staining (Fig. 1c).
Podoplanin was strongly positive on the cellular membranes of intercalating fibroblast-like cells and the inner surface of vessel-like clefts in the NFM (Fig. 1d).

\section{Histological staging of NFMs}

Histological observation revealed several stages of NFMs (Fig. 2), an early exudative phase (stage I), fibrosing phase (stage II), and late scarring phase (stage III). In the early exudative phase (stage I), fibrin was deposited, as detected by Masson trichrome staining (Fig. 2a) and confirmed by fibrin immunostaining (Fig. 2b). Podoplanin-positive fibroblasts were abundantly distributed in the NFM (Fig. 2c). In the fibrosing phase (stage II), fibrous matrices were dominant, with many collagen fibers and sparsely distributed fibroblast-like cells (Fig. 2d). In this stage, no fibrin deposition was detected immunohistochemically (Fig. 2e), whereas podoplanin was weakly positive in the fibroblast-like cells (Fig. 2f). In the scarring phase (stage III), the NFM was characterized by loose fibrous matrices covering the peritoneal surface and sometimes forming peritoneal adhesions with few cellular components (Fig. 2g). Neither fibrin (Fig. 2h) nor podoplanin (Fig. 2i) was detected in this phase. NFM stage was determined by Masson trichrome staining of fibrin and podoplanin or by immunohistochemistry (Fig. 2j). Other histological findings such as hemorrhage, inflammatory cell infiltration, and capillary proliferation were preferentially observed in

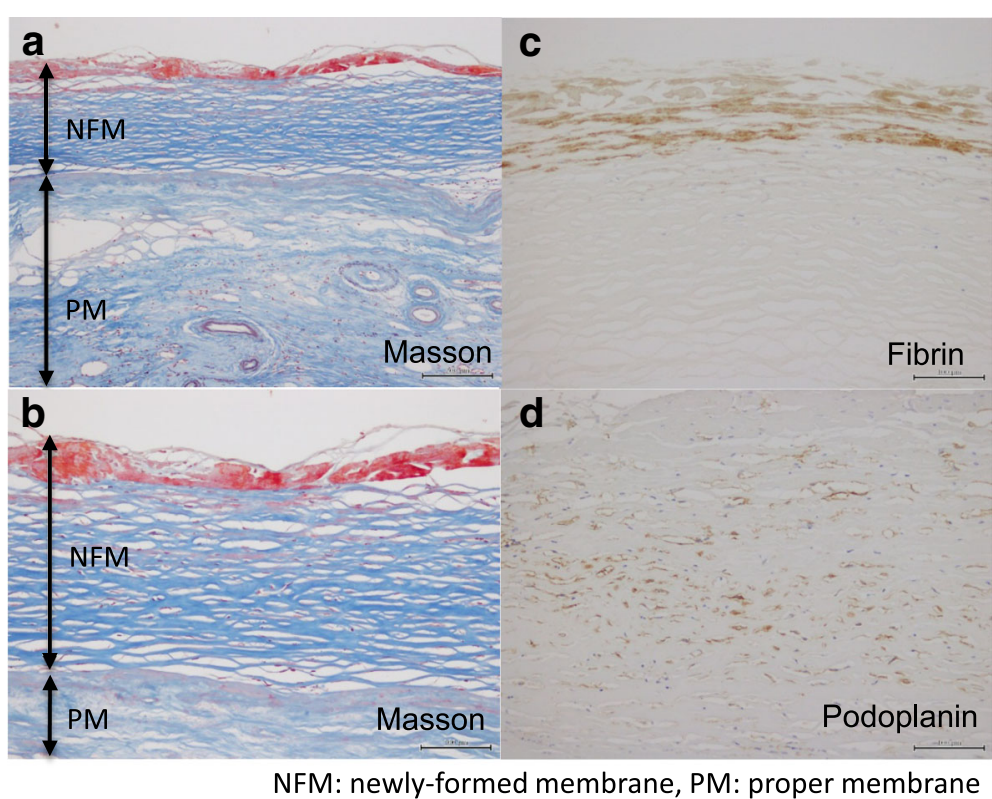

Fig. 1 Histological findings of an NFM in a representative EPS patient. An NFM is a fibrous membrane-like structure attached to the surface of the visceral peritoneum proper (PM). a The NFM is located on the visceral peritoneal membrane and has the histological appearance of simple peritoneal sclerosis with severe fibrosis and obstructive vasculopathy (Masson stain, $\times 100$, and bar $=200 \mu \mathrm{m}$ ). $\mathbf{b}$ The NFM is characterized by surface fibrin deposition and an interlacing of homogenous fibrous bundles with fibroblast-like cells (Masson stain, $\times 200$, and bar $=100 \mu \mathrm{m}$ ). c Fibrin is deposited on the surface of the NFM and confirmed by immunohistochemical fibrin staining (IHC for fibrin, $\times 200$, and bar $=100 \mu \mathrm{m}$ ). d Podoplanin is strongly positive on the cell membranes of intercalating fibroblast-like cells in the NFM (IHC for podoplanin, $\times 200$, and $\operatorname{bar}=100 \mu \mathrm{m})$ 


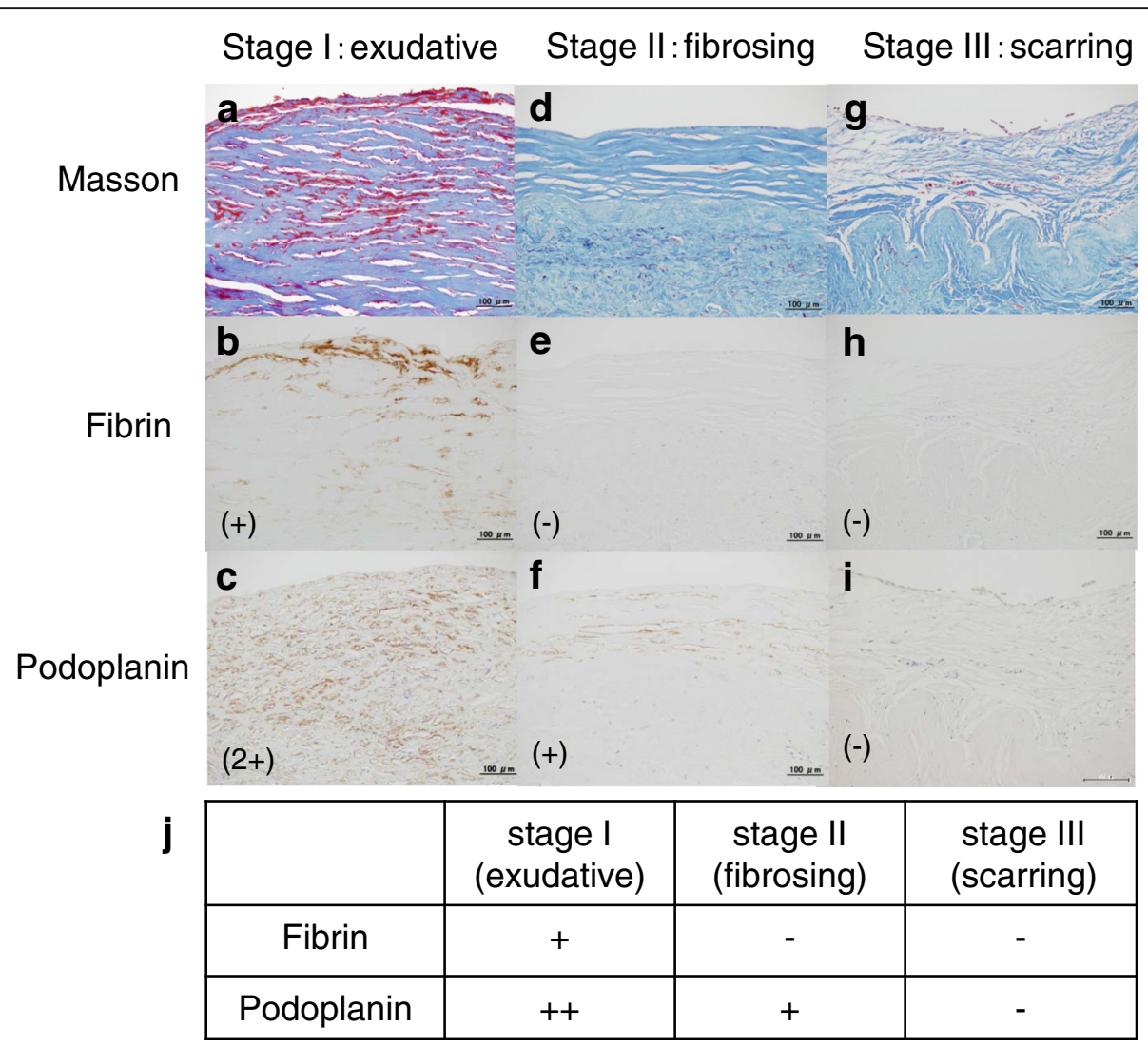

Fig. 2 Histological staging of NFMs. Histological observation revealed several stages of NFM. In the early exudative phase (stage I), fibrin deposition is apparent, as indicated by the bright red color in Masson trichrome staining (a) and confirmed by fibrin immunostaining (b). Podoplanin-positive fibroblasts are abundantly distributed in the encapsulating membrane (c). In the fibrotic phase (stage II), fibrosis is dominant, with many collagen fibers and sparsely distributed fibroblasts (d). In this stage, no fibrin deposition is detected immunohistochemically (e), whereas podoplanin is weakly positive in the fibroblast-like cells (f). In the scarring phase (stage III), the NFM is characterized by loose fibrous matrices covering the peritoneal surface, occasionally forming peritoneal adhesions with scant cellular components. Mesothelial cell coverage is observed on some areas of the peritoneal surface $(\mathbf{g})$. Both fibrin (h) and podoplanin (i) were no longer detected in this phase. NFM staging was determined by the detection of fibrin and podoplanin by Masson trichrome staining or immunohistochemistry (j). All figures are $\times 200$ and bars $=100 \mu \mathrm{m}$

the earlier stages (stages I and II). The mesothelial lining of the peritoneal surface was occasionally seen in later stages (stages II and III), but usually not in stage I.

\section{NFMs were observed in non-EPS patients}

NFMs were frequently observed in EPS patients and considered necessary for a diagnosis of EPS. However, NFMs were also identified microscopically in peritoneal samples of non-EPS patients, especially those with peritoneal lavage after PD discontinuation (Fig. 3). The NFM was usually identified histologically as a thin membrane on the peritoneal membrane proper and distinguished from the peritoneum proper by the presence of exudative features, swollen fibroblast-like cells, and absence of the expected vascular structure of arteries and veins present in the peritoneum proper (Fig. 3a, c, e). The detection of a layer of elastic fibers by Masson trichrome staining was helpful to distinguish the peritoneum proper from the new membrane because elastic fibers occur in the submesothelial zone of the peritoneal membrane but not in the NFM (Fig. 1a). Podoplanin immunostaining was also helpful to confirm the NFM because its expression in fibroblast-like cells was a characteristic finding in the NFM (Fig. 3b, d).

\section{Comparison of clinical and histological findings in EPS and non-EPS with or without peritoneal lavage}

All patients in the three groups were treated with conventional acidic PD solutions (Table 2). The nonEPS patients without lavage were younger (44.1 \pm 17.5 years of age) than both the EPS patients (56.7 \pm 5.7 years old) and the non-EPS patients with lavage (58.5 \pm 9.5 years old). The PD duration was longer in the EPS patients $(124.5 \pm 34.2$ months) than in both the non-EPS patients with lavage (94.8 \pm 33.7 months) and in those without lavage (91.4 \pm 30.3 months). Peritoneal lavage was performed in three of the EPS patients to prevent peritoneal adhesions before peritoneal dissecting surgery. 


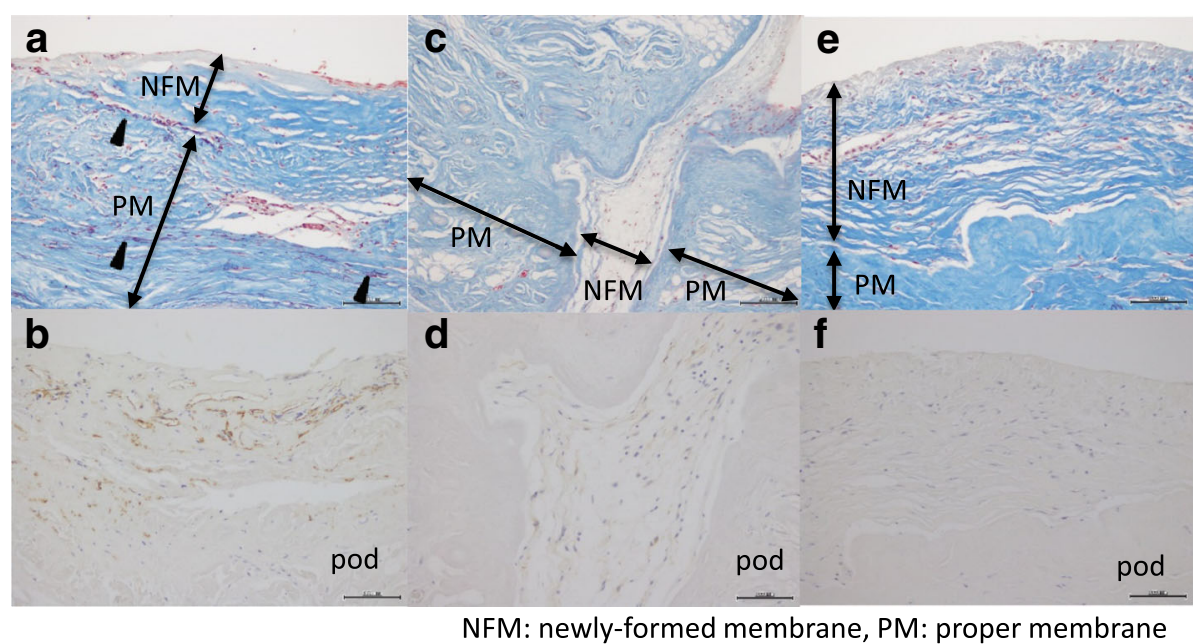

Fig. 3 Histological findings of NFMs in non-EPS patients. NFMs were observed microscopically in the peritoneal biopsies of non-EPS patients with peritoneal lavage treatment after completing PD. NFMs were attached to the peritoneal membrane proper and distinguished from the peritoneal membrane (PM) by the presence of exudative or fibrous features $(\mathbf{a}, \mathbf{c}, \mathbf{e})$. The presence of an elastic fiber layer detected by elastic-Masson trichrome staining was helpful to distinguish the NFM from the PM because the elastic fiber layer was located in the upper part of PM but not in the NFM (a). Podoplanin immunostaining was helpful to identify the NFM and determine its histological stage. Podoplanin expression in fibroblast-like cells is characteristic of the NFM (b, d). Negative podoplanin expression in fibroblast-like cells indicates stage III NFM (scarring phase) (e, f). Absence of the original vascular structure including artery and vein complexes is another index of NFM (a, $\mathbf{c}, \mathbf{e})$. NFMs in non-EPS patients shifted to later histological stages: stage II (seven cases) and stage III (seven cases), compared with NFMs in EPS patients: stage I (five cases), stage II (two cases), and stage III (one case). a Elastic-Masson staining, $\times 200$. c, e Masson staining, $\times 200$. b, d, f $1 H C$ for podoplanin, $\times 200$, bar $=100 \mu \mathrm{m}$

The category data of peritoneal equilibration test (PET) was available in 30 of non-EPS patients (16 with lavage and 14 without lavage), suggesting no difference between patients with and without peritoneal lavage.
Histological evaluation of the background peritoneum revealed that the average thickness of the SMC in EPS patients $(636.2 \pm 409.4 \mu \mathrm{m})$ and in the non-EPS patients with lavage $(603.8 \pm 386.6 \mu \mathrm{m})$ was greater than in nonEPS patients without lavage $(336.0 \pm 116.3 \mu \mathrm{m})$. It also

Table 2 Clinical and pathological characteristics of EPS patients $(n=10)$, non-EPS patients with lavage $(n=30)$, and non-EPS patients without lavage $(n=22)$

\begin{tabular}{|c|c|c|c|c|}
\hline & $\begin{array}{l}\text { EPS patients } \\
(n=10)\end{array}$ & $\begin{array}{l}\text { Non-EPS patients with } \\
\text { lavage }(n=30)\end{array}$ & $\begin{array}{l}\text { Non-EPS patients without } \\
\text { lavage }(n=22)\end{array}$ & $p$ value \\
\hline Age & $56.7 \pm 5.7 \neq$ & $58.5 \pm 9.5^{*}$ & $44.1 \pm 17.5^{*} \neq$ & $\begin{array}{l}<0.0041^{*} \\
<0.011 \neq \\
<0.0001(\mathrm{KW})\end{array}$ \\
\hline Gender & M5/F5 & M19/F11 & M13/F9 & NS (X2 test) \\
\hline PD duration (m) & $124.5 \pm 34.2^{*} \neq$ & $94.8 \pm 33.7^{*}$ & $91.4 \pm 30.3 \neq$ & $\begin{array}{l}<0.031^{*} \\
<0.021 \neq \\
<0.0001(\mathrm{KW})\end{array}$ \\
\hline Lavage (\%) & $3 / 10(30 \%)$ & $30 / 30(100 \%)$ & $0 / 22(0 \%)$ & ND \\
\hline PET category: H/HA/LA/L & ND & $4 / 7 / 4 / 1$ & $4 / 5 / 5 / 0$ & NS (X2 test) \\
\hline Average thickness of SMC ( $\mu \mathrm{m})$ & $636.2 \pm 409.4 \neq$ & $603.8 \pm 386.6^{*}$ & $336.0 \pm 116.3^{*} \neq$ & $\begin{array}{l}<0.0031^{*} \\
<0.05 \neq \\
0.063(\mathrm{KW})\end{array}$ \\
\hline Average $L / N$ ratio & $0.33 \pm 0.22 \ddagger$ & $0.39 \pm 0.20^{*}$ & $0.53 \pm 0.24^{*} \neq$ & $\begin{array}{l}<0.028^{*} \\
<0.031 \neq \\
0.048(\mathrm{KW})\end{array}$ \\
\hline Prevalence of NFM & $8 / 10(80 \%)$ & 13/30 (43.3\%) & $1 / 22(4.5 \%)$ & $\begin{array}{l}<0.0001 \\
(X 2 \text { test })\end{array}$ \\
\hline Stage of NFMs (I/II/III) & $5 / 2 / 1$ & $0 / 6 / 7$ & $0 / 1 / 0$ & ND \\
\hline
\end{tabular}


revealed that the average L/V in EPS patients $(0.33 \pm 0.22)$ was smaller than that of the non-EPS patients without lavage $(0.53 \pm 0.24)$. The average $\mathrm{L} / \mathrm{V}$ in the non-EPS patients with lavage $(0.39 \pm 0.20)$ was also smaller than that of the non-EPS patients without lavage. The decreased occurrence of peritoneal sclerosis in non-EPS patients without lavage might be explained by either treatment selection bias associated with peritoneal lavage after PD discontinuation or the younger age of that group. The frequency of NFM detection was significantly higher in EPS patients $(8 / 10,80 \%)$ than that in non-EPS patients with lavage $(13 / 30,43.3 \%)$ and those without lavage $(1 / 22$, $4.5 \%)$. The histological stages of NFM were predominantly earlier in EPS patients: stage I in five cases (62.5\%), stage II in two cases (25\%), and stage III in one case (12.5\%), and shifted to later stages in non-EPS patients: stage II and stage III in seven cases each (50\%) (Table 2). The comparison of the clinical and pathological characteristics of EPS patients $(n=10)$ and non-EPS patients $(n=52)$ was shown in Additional file 1: Table S1. The correlations between peritoneal sclerosis and PD duration in the three groups were represented in Additional file 1: Figure S1.

\section{Clinical and histological significance of new membrane formation in peritoneal biopsy tissue}

To investigate the clinical and histological significance of new membrane formation, we compared the biopsy data of cases with NFM $(n=13)$ and those without NFM $(n=17)$ in non-EPS patients with peritoneal lavage (Table 3). There were no significant differences of age, gender, PD duration, or post-PD duration (i.e., lavage duration) in the two groups. The PET category tended to be in high $(\mathrm{H})$ and high average (HA) in the patients with NFM, whereas shifted to be in low average (LA) and low (L) in those

Table 3 Clinical and histological significance of new membrane formation in peritoneal biopsies obtained at catheter removal in non-EPS patients with lavage $(n=30)$

\begin{tabular}{|c|c|c|c|}
\hline & $\begin{array}{l}\text { Non-EPS patients } \\
\text { with NFM }(n=13)\end{array}$ & $\begin{array}{l}\text { Non-EPS patients } \\
\text { without NFM } \\
(n=17)\end{array}$ & $p$ value \\
\hline Age & $58.2 \pm 11.1$ & $58.7 \pm 9.2$ & NS \\
\hline Gender & M9/F4 & M10/F7 & NS \\
\hline PD duration (m) & $91.9 \pm 18.8$ & $97.6 \pm 37.5$ & NS \\
\hline Post-PD duration (m) & $12.6 \pm 5.2$ & $12.9 \pm 5.4$ & NS \\
\hline $\begin{array}{l}\text { PET category: } \\
\text { H/HA/LA/L }\end{array}$ & $4 / 3 / 1 / 0$ & $0 / 4 / 3 / 1$ & NS (0.16) \\
\hline $\begin{array}{l}\text { Average thickness } \\
\text { of SMC }(\mu \mathrm{m})\end{array}$ & $560.8 \pm 315.5$ & $636.1 \pm 475.3$ & NS \\
\hline Average $L / N$ ratio & $0.33 \pm 0.12$ & $0.43 \pm 0.24$ & NS (0.16) \\
\hline Stage of NFM (I/II/III) & $0 / 6 / 7$ & 0/0/0 & ND \\
\hline
\end{tabular}

Mean \pm sd

PET peritoneal equilibration test, $H$ high, $H A$ high average, $L A$ low average, $L$ low, SMC submesothelial compact zone, LN lumen/vessel diameter, NFM newly formed membrane, NS not significant, ND not done without NFM, but the difference did not reach significance. Histologically, the average SMC thickness and L/V ratio were not significantly different in the two groups; the $\mathrm{L} / \mathrm{V}$ ratio in patients with NFM was lower than that in patients without new membranes $(0.33 \pm 0.12$ vs. $0.43 \pm 0.24$, $p=0.16$ ) but the difference did not reach significance. The clinical and histological relevance of histological staging of NFMs in EPS patients $(n=8)$ and in all patients with NFMs in the three groups $(n=22)$ was shown in Additional file 1: Tables S2 and S3, respectively.

\section{Discussion}

\section{Definition of NFM and its histological features}

Encapsulation in EPS is caused by an NFM on the peritoneal membrane proper, which can be distinguished by careful macroscopic and microscopic observation. Surgeon can usually identify the NFM by peeling it away from the intestinal serosa during enterolysis surgery. Laparoscopic observation can also confirm the NFM by its unique cocoon-like appearance over the gastroenteric organs. The histological appearance of NFMs resembles to that of fibrotic regions of simple peritoneal sclerosis; however, the NFM can be distinguished by the absence of large vessels, nerves, and adipose tissue that are usually observed in the peritoneum proper.

Various histological features and the stages of NFMs are suggestive of the initiation and progression of EPS. At early stages, NFMs are primarily composed of exudative fibrin, suggesting that the exudation of plasma containing fibrin/fibrinogen and other blood coagulation factors is required for encapsulating membrane formation [17]. The cocoonlike encapsulating membrane recognized macroscopically during the enterolysis surgery is usually characterized histologically by exudative fibrin and its organization and consistent with NFM in stage I. Increased permeability of the peritoneal membrane, a very common complication of long-term PD [18-20], may be an important background factor for the pathogenesis of EPS. Increased peritoneal vascularity is thought to be associated with the peritoneal hyperpermeability observed in the previous histological studies of PD peritoneum [21-23]. Recently, Tawada et al. reported that damage to vascular endothelium, represented by hyalinizing vasculopathy of the peritoneal membrane, was predictive of the development of EPS and suggested that peritoneal hyperpermeability could result from vascular endothelial injury [24]. Morelle et al. demonstrated that an early, relatively large reduction in osmotic conductance (sodium sieving) during PD was an independent predictor of EPS and that such functional disorders were associated with the extent of peritoneal fibrosis, but not with vasculopathy [25]. Although the precise mechanism of peritoneal hyperpermeability in long-term PD patients is still controversial, disturbance of peritoneal membrane transport across the peritoneal microvasculature has a significant role in the pathogenesis of EPS. 
This study also identified differences in the histological stages of NFM in different clinical situations. Early stages of NFM were frequently observed in EPS patients who experienced enterolysis surgery. On the other hand, later stages of NFM were seen in non-EPS patients with peritoneal lavage after completing PD. This difference could be associated with temporal phase of inflammatory process, showing active peritoneal injury in EPS patients with enterolysis surgery and chronic subsiding peritoneal damage in non-EPS patients with peritoneal lavage for several months after completion of PD. Therefore, the fresh fibrin deposition in histological stage I may be a possible diagnostic criterion for EPS in acute/active phase and it may disappear in chronic/subsiding phase. The predominance of early stages in EPS patients might be associated with a bias of biopsy sampling from visceral peritoneum. There may be some pathophysiological differences between visceral and parietal peritoneum, although they have not been fully established so far. Yaginuma et al. demonstrated increased lymphatic vessels in visceral peritoneum rather than parietal peritoneum [26], and this difference might be related to the susceptibility of encapsulating membrane formation in visceral peritoneum via increased exudation. The histological stage may also indicate the consequences of NFMs, that is progression to encapsulation and adhesion or scar formation without the development of EPS.

Significance of podoplanin expression in new membranes This study confirmed the utility of podoplanin staining in the pathological diagnosis and staging of EPS. Braun et al. first reported that the presence podoplaninpositive fibroblast-like cells was a good marker of EPS $[11,12]$. Podoplanin was first described as a molecule expressed on glomerular podocytes [27] and was later was found to be a useful marker of lymphatic endothelial [28] and mesothelial cells [29]. Recent investigations revealed that podoplanin is a transmembranous glycoprotein whose ligand is C-type lectin receptor-2 (CLEC2) on platelets and inflammatory cells [30]. This protein is involved in cell motility, migration, and proliferation during development, in the immune system, and in cancer [31]. Because EPS develops in association with increased accumulation of serum and high concentrations of fibrin [17, 32], secretion of cytokines and growth factors [26, 33-35] may activate the transformation of peritoneal mesenchymal cells into swollen fibroblast-like cells that highly express podoplanin on their cell membranes. Podoplanin-positive fibroblast-like cells in the peritoneum of EPS patients may be activated by platelets and inflammatory cells expressing podoplanin-ligand, CLEC-2 [30], and by the surrounding extracellular matrix containing hyaluronic acid [31]. Thus, podoplanin expression may be associated with the disease activity or the histological stage of EPS.

\section{NFMs were detected in non-EPS patients}

To evaluate the diagnostic specificity of the NFM in EPS, we investigated the prevalence of NFM in the peritoneal biopsy specimens obtained from EPS patients at enterolysis surgery and in non-EPS patients at catheter removal. NFMs were detected in most of the EPS patients (8/10 cases, $80 \%$ ), and although biopsies were not efficient for the detection of NFM in the other two patients, NFMs encapsulating the intestines had been macroscopically confirmed during enterolysis surgery. Therefore, it is reasonable to conclude that the detection of NFM, either macroscopically or microscopically, is required for a pathological diagnosis of EPS. On the other hand, NFMs were also detected microscopically in non-EPS patients (14/52 cases, 26.9\%) suggesting that microscopic NFMs could not predict the development of EPS and was not a sufficient condition for the diagnosis of EPS. A similar result has been previously reported in a study by Sherif et al. comparing the peritoneal pathology of EPS and non-EPS patients, in which NFMs were observed in 6/23 non-EPS patients (26.1\%) [8]. In an evaluation of new membrane formation in peritoneal biopsies at catheter removal, Tawada et al. also observed the presence of NFMs in non-EPS patients and found increased histological scores of NFMs in EPS patients, but the difference in comparison with non-EPS patients did not reach significance [24]. Because new membrane formation is a result of increased exudation on the peritoneal surface, the detection of NFM suggests the increased permeability of the peritoneal vessels. The increased incidence of NFMs in the peritoneal lavage group suggested that new membrane formation occurred primarily during the period of lavage after completion of PD. Nevertheless, the detection of NFMs did not predict the subsequent development of EPS in this study, indicating that microscopically detected NFMs probably regressed and did not progress to encapsulation of abdominal organs.

\section{Role of peritoneal sclerosis in new membrane formation} We also evaluated pathological differences of the background peritoneum in EPS patients and non-EPS patients. The results indicated a tendency for an advanced grade of peritoneal vasculopathy in the EPS patients. These observations support previous observations that prolonged PD duration was the most important risk factor for EPS [18, 36-38] and that histological damage of the peritoneum is closely associated with high peritoneal transport, which is another risk factor for EPS $[19,20]$. Increased microvascular density of the background peritoneum proper is thought to be associated with hyperpermeability of the peritoneal membrane and to promote EPS development [21-23]. However, we observed no significant difference of microvascular density in the peritoneum proper in EPS patients and non-EPS patients (data not shown). Further investigation is required to elucidate the functional and morphological relationships of microvascular density and peritoneal hyperpermeability in the pathogenesis of EPS. 
Vascular density may not necessarily be associated with increased peritoneal permeability and subsequent formation of encapsulating membranes. The nonphysiological state of the vascular barrier, an endothelial layer of the peritoneal capillary, may be associated with impaired peritoneal barrier function observed with long-term PD treatment [39-41]. Functional deterioration of peritoneal microvascular permeability induced by PD, rather than morphological damages, might be associated with the onset of EPS and should be considered for understanding the pathogenesis of EPS.

\section{Effect of neutral, low glucose degradation product (GDP) dialysate on peritoneal sclerosis, and EPS}

Since biocompatible PD solutions with neutral $\mathrm{pH}$ and low GDP have become available, the incidence and extent of PD-induced peritoneal deterioration, characterized as simple peritoneal sclerosis, has decreased [42-44]. As simple peritoneal sclerosis is the most important risk factor for EPS, the incidence of EPS is expected to decrease. A recent report has shown a decrease in EPS incidence (1.0\%) in Japan [45]. This tendency suggests that the use of biocompatible PD solution is the most effective, and indispensable, strategy for the prevention of EPS. The merits of biocompatible PD solutions are probably attributable to its low GDP concentration, which is the component with the highest toxicity for cellular and extracellular matrix components such as collagen fibers [46]. Peritoneal permeability is primarily determined by the capillary endothelium [41]. Preservation of the physiological integrity of peritoneal tissue contributes to vascular integrity and prevents increased permeability of the peritoneal membrane, a key factor in encapsulating membrane formation.

\section{Limitations of the present study}

Some limitations must be considered when interpreting the study results. First, this was a multicenter retrospective observational study, and not all the patients with PD withdrawal were included, thus there must be a selection bias in each cohort. Second, the clinical information was limited, and many factors like the daily ultrafiltration volume, the peritoneal equilibration test, the amount of glucose exposure, and the method of peritoneal lavage could not be evaluated. Third, the number of patients in each group limited the power of the statistical comparisons. Fourth, the long-term follow-up was not available to determine the clinical outcome, such as the development of EPS. Fifth, the method of peritoneal biopsy was not standardized, so the quality and quantity of the biopsy samples were not always adequate for histological evaluation. Sixth, there was a sampling bias of biopsy materials, which were taken from visceral peritoneum in most of the EPS patients (8/10) and from parietal in all of the non-EPS patients $(n=52)$. It might have some influences on the staging of NFMs and predominance of stage I in the EPS patients. The above limitations may be associated with some inconclusive results. Nevertheless, the study reports important evidence of the appearance of NFM in peritoneal biopsy samples from both EPS patients and non-EPS patients.

\section{Conclusions}

The pathological diagnosis of EPS requires confirmation of NFM, either macroscopically or microscopically, which is a strong pathological hallmark of EPS. However, NFMs were detected microscopically in non-EPS patients, especially those with peritoneal lavage after completion of PD discontinuation. New membrane formation, which is believed to be an early pathological phenomenon of EPS, reflects increased exudation from peritoneal vasculature, associated with the initiation of, but does not always predict, the development of EPS.

\section{Additional file}

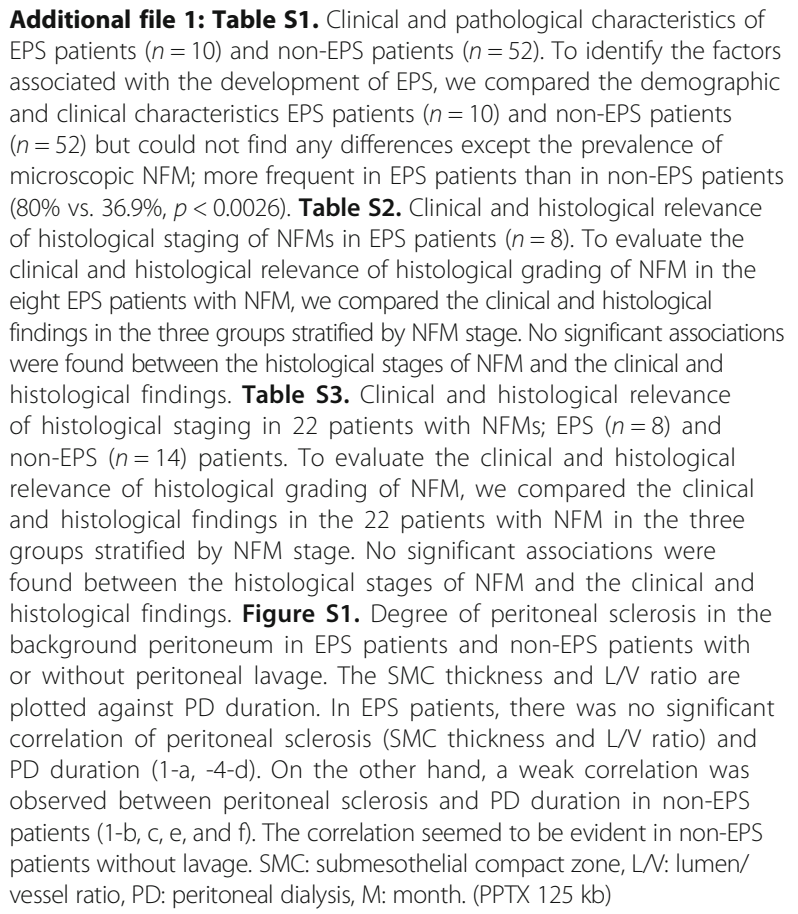
associated with the development of EPS, we compared the demographic and clinical characteristics EPS patients $(n=10)$ and non-EPS patients ( $n=52$ ) but could not find any differences except the prevalence of microscopic NFM; more frequent in EPS patients than in non-EPS patients ( $80 \%$ vs. $36.9 \%, p<0.0026$ ). Table S2. Clinical and histological relevance of histological staging of NFMs in EPS patients $(n=8)$. To evaluate the clinical and histological relevance of histological grading of NFM in the eight EPS patients with NFM, we compared the clinical and histological findings in the three groups stratified by NFM stage. No significant associations were found between the histological stages of NFM and the clinical and histological findings. Table S3. Clinical and histological relevance of histological staging in 22 patients with NFMs; EPS $(n=8)$ and non-EPS $(n=14)$ patients. To evaluate the clinical and histological relevance of histological grading of NFM, we compared the clinical and histological findings in the 22 patients with NFM in the three groups stratified by NFM stage. No significant associations were found between the histological stages of NFM and the clinical and histological findings. Figure S1. Degree of peritoneal sclerosis in the background peritoneum in EPS patients and non-EPS patients with or without peritoneal lavage. The SMC thickness and $L / N$ ratio are plotted against PD duration. In EPS patients, there was no significant correlation of peritoneal sclerosis (SMC thickness and LN ratio) and PD duration (1-a, -4-d). On the other hand, a weak correlation was observed between peritoneal sclerosis and PD duration in non-EPS patients (1-b, c, e, and f). The correlation seemed to be evident in non-EPS patients without lavage. SMC: submesothelial compact zone, LN: lumen/ vessel ratio, PD: peritoneal dialysis, M: month. (PPTX $125 \mathrm{~kb}$ )

\section{Abbreviations \\ EPS: Encapsulating peritoneal sclerosis; LN ratio: Lumen/vessel ratio; NFM: Newly formed membrane; PCV: Post-capillary venule; PD: Peritoneal dialysis; SMC: Submesothelial compact zone}

\section{Acknowledgements}

The authors would like to express their special thanks to Dr. Hiroshi Hirano for his significant contribution to the Peritoneal Biopsy Program in Japan. The authors also thank all the clinical physicians who participated in this program. The authors would like to thank Enago (www.enago.com) for the English language review. 


\section{Funding}

The Peritoneal Biopsy Program in Japan conducted from 1994 to 2006 was financially supported by Baxter Japan Co. Ltd. The funder had no role in this study design, data collection and analysis, decision to publish, or preparation of the manuscript.

\section{Availability of data and materials}

Not applicable.

\section{Authors' contributions}

$\mathrm{KH}$ and $\mathrm{KK}$ performed the histological examination of peritoneal biopsy tissue and analyzed and interpreted the clinical and histological data. $\mathrm{CH}$ and MN analyzed and interpreted the clinical and histological data. MM and $\mathrm{Yl}$ contributed to interpretation of the data and writing of the manuscript. JSPD organized the Peritoneal Pathology Study Committee and supported the activity. All authors read and approved the final manuscript.

\section{Competing interests}

The authors, except YI, declare that they have no competing interests regarding this article. $\mathrm{YI}$ is a professor in endowed chair supported by Baxter Japan Co. Ltd.

\section{Consent for publication}

Not applicable.

\section{Ethics approval and consent to participate}

The study design was approved by the ethics committee of Juntendo Medical University (No. 370, May 2009). Written informed consent was obtained from all study subjects.

\section{Publisher's Note}

Springer Nature remains neutral with regard to jurisdictional claims in published maps and institutional affiliations.

\section{Author details \\ 'Department of Anatomy, Showa University School of Medicine, 1-5-8 Hatanodai, Shinagawa-ku, Tokyo 142-8555, Japan. ²Division of Nephrology, Juntendo Medical University Faculty of Medicine, 2-1-1 Hongo, Bunkyo-ku, Tokyo 113-8421, Japan. ${ }^{3}$ Department of Surgical Pathology, Tokyo Women's Medical University, 8-1 Kawada-cho, Shinjuku-ku, Tokyo 162-8666, Japan. ${ }^{4}$ Research Division of Chronic Kidney Disease and Dialysis Treatment, Tohoku University Hospital, 1-1 Seiryo-cho, Aoba-ku, Sendai-shi, Miyagi 980-0872, Japan. ${ }^{5}$ Miyazaki Clinic, 3-12 Shiratori-cho, Nagasaki-shi, Nagasaki 852-8042, Japan. ${ }^{6}$ Department of Nephrology and Renal Replacement Therapy, Nagoya University Graduate School of Medicine, 65 Tsurumai-cho, Showa-ku, Nagoya 466-8550, Japan. ${ }^{7}$ Japanese Society of Peritoneal Dialysis (JSPD), 1-39 Kitasakoichiban-cho, Tokushima-shi, Tokushima 770-0011, Japan.}

\section{Received: 20 January 2017 Accepted: 21 April 2017}

\section{Published online: 09 August 2017}

\section{References}

1. Gandhi VC, Humayun HM, Ing TS, Daugirdas JT, Jablokow VR, Iwatsuki S, Geis WP, Hano JE. Sclerotic thickening of the peritoneal membrane in maintenance peritoneal dialysis patients. Arch Intern Med. 1980;140:1201-3.

2. Dobbie JW. Pathogenesis of peritoneal fibrosing syndromes (sclerosing peritonitis) in peritoneal dialysis. Perit Dial Int. 1992;12:14-27.

3. Nomoto Y, Kawaguchi Y, Kubo H, Hirano H, Sakai S, Kurokawa K. Sclerosing encapsulating peritonitis in patients undergoing continuous ambulatory peritoneal dialysis: a report of the Japanese Sclerosing Encapsulating Peritonitis Study Group. Am J Kidney Dis. 1996;28:420-7.

4. Kawaguchi Y, Kawanishi H, Mujais S, Topley N, Oreopoulos DG. Encapsulating peritoneal sclerosis: definition, etiology, diagnosis, and treatment. International Society for Peritoneal Dialysis Ad Hoc Committee on Ultrafiltration Management in Peritoneal Dialysis. Perit Dial Int. 2000;20 (Suppl 4):S43-55.

5. Kawaguchi $Y$, Saito A, Kawanishi $H$, Nakayama M, Miyazaki M, Nakamoto $H$, Tranaeus A. Recommendations on the management of encapsulating peritoneal sclerosis in Japan, 2005: diagnosis, predictive markers, treatment, and preventive measures. Perit Dial Int. 2005;25 (Suppl 4):S83-95.
6. Honda K, Oda H. Pathology of encapsulating peritoneal sclerosis. Perit Dial Int. 2005;25 (Suppl 4):S19-29.

7. Honda K, Nitta K, Horita S, Tsukada M, Itabashi M, Nihei H, Akiba T, Oda H. Histologic criteria for diagnosing encapsulating peritoneal sclerosis in continuous ambulatory peritoneal dialysis patients. Adv Perit Dial. 2003;19: 169-75.

8. Sherif AM, Yoshida H, Maruyama Y, Yamamoto H, Yokoyama K, Hosoya T, Kawakami M, Nakayama M. Comparison between the pathology of encapsulating sclerosis and simple sclerosis of the peritoneal membrane in chronic peritoneal dialysis. Ther Apher Dial. 2008;12:33-41.

9. Braun N, Fritz P, Ulmer C, Latus J, Kimmel M, Biegger D, Ott G, Reimold F, Thon KP, Dippon J, Segerer S, Alscher MD. Histological criteria for encapsulating peritoneal sclerosis - a standardized approach. PLoS ONE. 2012;7:e48647.

10. Latus J, Ulmer C, Fritz P, Rettenmaier B, Biegger D, Lang T, Ott G, Kimmel M, Steurer W, Alscher MD, Segerer S, Braun N. Phenotypes of encapsulating peritoneal sclerosis - macroscopic appearance, histologic findings, and outcome. Perit Dial Int. 2013;33:495-502.

11. Braun N, Alscher DM, Fritz P, Edenhofer I, Kimmel M, Gaspert A, Reimold F, Bode-Lesniewska B, Ziegler U, Biegger D, Wüthrich RP, Segerer S. Podoplanin-positive cells are a hallmark of encapsulating peritoneal sclerosis. Nephrol Dial Transplant. 2011;26:1033-41.

12. Braun N, Alscher MD, Fritz P, Latus J, Edenhofer I, Reimold F, Alper SL, Kimmel M, Biegger D, Lindenmeyer M, Cohen CD, Wüthrich RP, Segerer S. The spectrum of podoplanin expression in encapsulating peritoneal sclerosis. PLoS ONE. 2012;7:e53382.

13. Yamamoto T, Nagasue K, Okuno S, Yamakawa T. The role of peritoneal lavage and the prognostic significance of mesothelial cell area in preventing encapsulating peritoneal sclerosis. Perit Dial Int. 2010;30:343-52.

14. Ubara Y, Tagami T, Hara S. Successful peritoneal lavage therapy for prevention of encapsulating peritoneal sclerosis: a case report. Ther Apher Dial. 2011;15:211-3.

15. Brown EA, Van Biesen W, Finkelstein FO, Hurst $H$, Johnson DW, Kawanishi $H$, Pecoits-Filho R, Woodrow G, ISPD Working Party. Length of time on peritoneal dialysis and encapsulating peritoneal sclerosis: position paper for ISPD. Perit Dial Int. 2009;29:595-600.

16. Honda K, Hamada C, Nakayama M, Miyazaki M, Sherif AM, Harada T, Hirano $\mathrm{H}$, Peritoneal Biopsy Study Group of the Japanese Society for Peritoneal Dialysis. Impact of uremia, diabetes, and peritoneal dialysis itself on the pathogenesis of peritoneal sclerosis: a quantitative study of peritoneal membrane morphology. Clin J Am Soc Nephrol. 2008;3:720-8.

17. Dobbie JW, Jasani MK. Role of imbalance of intracavity fibrin formation and removal in the pathogenesis of peritoneal lesions in CAPD. Perit Dial Int. 1997:17:121-4.

18. Yamamoto R, Otsuka Y, Nakayama M, Maruyama Y, Katoh N, Ikeda M, Yamamoto H, Yokoyama K, Kawaguchi Y, Matsushima M. Risk factors for encapsulating peritoneal sclerosis in patients who have experienced peritoneal dialysis treatment. Clin Exp Nephrol. 2005;9:148-52.

19. Habib AM, Preston E, Davenport A. Risk factors for developing encapsulating peritoneal sclerosis in the icodextrin era of peritoneal dialysis prescription. Nephrol Dial Transplant. 2010;25:1633-8.

20. Lambie ML, John B, Mushahar L, Huckvale C, Davies SJ. The peritoneal osmotic conductance is low well before the diagnosis of encapsulating peritoneal sclerosis is made. Kidney Int. 2010;78:611-8.

21. Mateijsen MA, van der Wal AC, Hendriks PM, Zweers MM, Mulder J, Struijk DG, Krediet RT. Vascular and interstitial changes in the peritoneum of CAPD patients with peritoneal sclerosis. Perit Dial Int. 1999;19:517-25.

22. Numata M, Nakayama M, Nimura S, Kawakami M, Lindholm B, Kawaguchi Y. Association between an increased surface area of peritoneal microvessels and a high peritoneal solute transport rate. Perit Dial Int. 2003;23:116-22.

23. Sherif AM, Nakayama M, Maruyama Y, Yoshida H, Yamamoto H, Yokoyama K Kawakami M. Quantitative assessment of the peritoneal vessel density and vasculopathy in CAPD patients. Nephrol Dial Transplant. 2006;21:1675-81.

24. Tawada M, Ito Y, Hamada C, Honda K, Mizuno M, Suzuki Y, Sakata F, Terabayashi T, Matsukawa Y, Maruyama S, Imai E, Matsuo S, Takei Y. Vascular endothelial cell injury is an important factor in the development of encapsulating peritoneal sclerosis in long-term peritoneal dialysis patients. PLoS ONE. 2016;11:e0154644.

25. Morelle J, Sow A, Hautem N, Bouzin C, Crott R, Devuyst O, Goffin E. Interstitial fibrosis restricts osmotic water transport in encapsulating peritoneal sclerosis. J Am Soc Nephrol. 2015;26:2521-33. 
26. Yaginuma T, Yamamoto I, Yamamoto H, Mitome J, Tanno Y, Yokoyama K, Hayashi T, Kobayashi T, Watanabe M, Yamaguchi Y, Hosoya T. Increased lymphatic vessels in patients with encapsulating peritoneal sclerosis. Perit Dial Int. 2012;32:617-27.

27. Breiteneder-Geleff S, Matsui K, Soleiman A, Meraner P, Poczewski H, Kalt R, Schaffner G, Kerjaschki D. Podoplanin, novel 43-kd membrane protein of glomerular epithelial cells, is down-regulated in puromycin nephrosis. Am J Pathol. 1997;151:1141-52.

28. Wetterwald A, Hoffstetter W, Cecchini MG, Lanske B, Wagner C, Fleisch H, Atkinson M. Characterization and cloning of the E11 antigen, a marker expressed by rat osteoblasts and osteocytes. Bone. 1996;18:125-32.

29. Kimura N, Kimura I. Podoplanin as a marker for mesothelioma. Pathol Int. 2005;55:83-6.

30. Suzuki-Inoue K, Kato Y, Inoue O, Kaneko MK, Mishima K, Yatomi Y, Yamazaki $Y$, Narimatsu $H$, Ozaki $Y$. Involvement of the snake toxin receptor CLEC-2, in podoplanin-mediated platelet activation, by cancer cells. J Biol Chem. 2007; 282:25993-6001.

31. Astarita JL, Acton SE, Turley SJ. Podoplanin: emerging functions in development, the immune system, and cancer. Front Immunol. 2012;3:283.

32. Fang CC, Huang JW, Shyu RS, Yen CJ, Shiao CH, Chiang CK, Hu RH, Tsai TJ. Fibrin-Induced epithelial-to-mesenchymal transition of peritoneal mesothelial cells as a mechanism of peritoneal fibrosis: effects of pentoxifylline. PLoS ONE. 2012;7:e44765.

33. Patel P, West-Mays J, Kolb M, Rodrigues JC, Hoff CM, Margetts PJ. Platelet derived growth factor $B$ and epithelial mesenchymal transition of peritoneal mesothelial cells. Matrix Biol. 2010;29:97-106.

34. Pérez-Lozano ML, Sandoval P, Rynne-Vidal A, Aguilera A, Jiménez-Heffernan JA, Albar-Vizcaíno P, Majano PL, Sánchez-Tomero JA, Selgas R, LópezCabrera M. Functional relevance of the switch of VEGF receptors/coreceptors during peritoneal dialysis-induced mesothelial to mesenchymal transition. PLOS ONE. 2013;8:e60776.

35. Kinashi $H$, Ito $Y$, Mizuno M, Suzuki $Y$, Terabayashi T, Nagura F, Hattori R, Matsukawa Y, Mizuno T, Noda Y, Nishimura H, Nishio R, Maruyama S, Imai E, Matsuo S, Takei Y. TGF- $\beta 1$ promotes lymphangiogenesis during peritoneal fibrosis. J Am Soc Nephrol. 2013;24:1627-42.

36. Kawanishi H, Kawaguchi Y, Fukui H, Hara S, Imada A, Kubo H, et al. Encapsulating peritoneal sclerosis in Japan: a prospective, controlled, multicenter study. Am J Kidney Dis. 2004;44:729-37.

37. Brown MC, Simpson K, Kerssens JJ, Mactier RA, Scottish Renal Registry. Encapsulating peritoneal sclerosis in the new millennium: a national cohort study. Clin J Am Soc Nephrol. 2009;4:1222-9.

38. Johnson DW, Cho Y, Livingston BE, Hawley CM, McDonald SP, Brown FG, Rosman JB, Bannister KM, Wiggins KJ. Encapsulating peritoneal sclerosis: incidence, predictors, and outcomes. Kidney Int. 2010;77:904-12.

39. Davies SJ. Peritoneal solute transport-we know it is important, but what is it? Nephrol Dial Transplant. 2000;15:1120-3.

40. Flessner MF. Endothelial glycocalyx and the peritoneal barrier. Perit Dial Int. 2008;28:6-12.

41. Rippe B, Davies S. Permeability of peritoneal and glomerular capillaries: what are the differences according to pore theory? Perit Dial Int. 2011;31:249-58.

42. Ayuzawa N, Ishibashi Y, Takazawa Y, Kume H, Fujita T. Peritoneal morphology after long-term peritoneal dialysis with biocompatible fluid: recent clinical practice in Japan. Perit Dial Int. 2012;32:159-67.

43. Kawanishi K, Honda K, Tsukada M, Oda H, Nitta K. Neutral solution low in glucose degradation products is associated with less peritoneal fibrosis and vascular sclerosis in patients receiving peritoneal dialysis. Perit Dial Int. 2013; 33:242-51.

44. Hamada C, Honda K, Kawanishi K, Nakamoto H, Ito Y, Sakurada T, Tanno Y, Mizumasa T, Miyazaki M, Moriishi M, Nakayama M. Morphological characteristics in peritoneum in patients with neutral peritoneal dialysis solution. J Artif Organs. 2015;18:243-50.

45. Nakayama M, Miyazaki M, Honda K, Kasai K, Tomo T, Nakamoto H, Kawanishi $H$. Encapsulating peritoneal sclerosis in the era of a multi-disciplinary approach based on biocompatible solutions: the NEXT-PD study. Perit Dial Int. 2014;34:766-74.

46. Cho Y, Johnson DW, Badve SV, Craig JC, Strippoli GF, Wiggins KJ. The impact of neutral-pH peritoneal dialysates with reduced glucose degradation products on clinical outcomes in peritoneal dialysis patients. Kidney Int. 2013;84:969-79.

\section{Submit your next manuscript to BioMed Central and we will help you at every step:}

- We accept pre-submission inquiries

- Our selector tool helps you to find the most relevant journal

- We provide round the clock customer support

- Convenient online submission

- Thorough peer review

- Inclusion in PubMed and all major indexing services

- Maximum visibility for your research

Submit your manuscript at www.biomedcentral.com/submit 